\title{
Un modelo multifractal simplificado para flujos de tráfico autosimilares
}

\section{A simplified multifractal model for self-similar traffic flows}

\author{
Ginno Millán \\ Universidad Católica del Norte, Larrondo 1281, Coquimbo, Chile
}

DOI: https://doi.org/10.33017/RevECIPeru2014.0003/

\begin{abstract}
Resumen
Este artículo propone un nuevo modelo multifractal, con el ánimo de proveer una posible explicación al fenómeno de localidad que aparece en la estimación del exponente de Hurst en series temporales estacionarias de segundo orden, representativas de los flujos de tráfico autosimilares en las actuales redes de computadoras de alta velocidad. Analíticamente se demuestra que este fenómeno se presenta cuando los flujos se componen de diversos tipos de tráficos con diferentes exponentes de Hurst.
\end{abstract}

Descriptores: Autosimilitud, exponente de Hurst (H), fenómeno de localidad, multifractales.

\begin{abstract}
This paper proposes a new multifractal model, with the aim to provide a possible explanation to the locality phenomena to appear in the estimation of Hurst exponent in stationary second order temporal series, representing the self-similar traffic flows in high-speed computer networks. Analytically it is shown that this phenomenon occurs if the network flow consists of several components whit different Hurst exponents.
\end{abstract}

Keywords: Self-similarity, Hurst exponent $(H)$, locality phenomena, multifractals.

\section{Introducción}

Las propiedades que evidencian la naturaleza de origen fractal de los flujos de tráfico en las redes de computadoras de alta velocidad, es un tema que ha sido ampliamente reportado en la literatura durante las últimas dos décadas; constituyendo una opinión generalizada el hecho de que su comportamiento dinámico re-escalado debe ser cuidadosamente atendido en los análisis de rendimiento y control. Existen, de esta forma, numerosas explicaciones y modelos que intentan entregar una respuesta a este origen [1]-[3].

Por otra parte, reconociendo que las localidades de un proceso fractal solo pueden ser analizadas desde la óptica del análisis multifractal; como producto de su construcción a partir de cascadas multiplicativas [4] que aseguran una caracterización más exacta producto del análisis a altas frecuencias [5], se acepta que los flujos de tráfico presentes en las actuales redes de computadoras de alta velocidad poseen naturaleza multifractal, lo cual da origen a un nuevo modelo de flujos que pretende explicar el fenómeno de localidad presente en la estimación del exponente de Hurst [3], [5].

A partir de los resultados obtenidos mediante el uso de simulaciones computacionales en MATLAB, se infiere que el modelo contribuye al conocimiento de la dinámica real del tráfico en las actuales redes de computadoras de alta velocidad, y puede ser utilizado para simular de manera simple tráfico real.

\section{Tráfico autosimilar y tráfico multifractal}

En las redes de computadoras los flujos de tráfico se consideran a menudo representados mediante un proceso autosimilar $Y(k)$ que satisface [6] 


$$
Y(k)={ }_{d} a^{-H} Y(a k), \quad \forall a>0, k \geq 0
$$

donde $=_{d}$ representa igualdad de distribuciones finito dimensionales y $H \in(0,1)$ es el exponente de Hurst del proceso estocástico autosimilar (H-SS) $Y(k)$.

Un proceso $\mathrm{H}$-SS con incrementos estacionarios descrito en términos del comportamiento de sus agregaciones, se obtiene de la multiplexación de incrementos $X(k)=Y(k+1)-Y(k)$ sobre bloques no superpuestos de tamaño $n$ según

$$
X^{(n)}(k)=\sum_{j=0}^{n-1} X(k n-j), \quad k \in \square, n \in \square
$$

El proceso $X^{(n)}(k)$ resultante tiene distribuciones finito dimensionales similares a las de $X(k)$, es decir

$$
X^{(n)}(k)={ }_{d} n^{H} X(k), \quad n \in \square
$$

El proceso estacionario $X(k)$ que cumple con (3) se llama proceso estacionario autosimilar, H-SSS, con exponente de Hurst $H$. Un típico ejemplo es el Ruido Fraccional Gaussiano (FGN), el cual viene dado por la expresión $X(k)=B^{(H)}(k+1)-B^{(H)}(k)[5]$.

Existen varias formas de estudiar las propiedades estadísticas de $X^{(n)}(k)$. En [5] se consideran los cumulantes de las series agregadas, los cuales se definen en términos de los coeficientes de Taylor de la función generatriz de cumulantes

$$
g(t)=\log \mathrm{E}\left(e^{t X(k)}\right)=\sum_{m=1}^{\infty} t^{m} m !^{-1} \operatorname{cum}_{m} X(k)
$$

donde $\operatorname{cum}_{m} X(k)=g^{(m)}(0)$. En [7] se demuestra que los cumulantes de $m$-ésimo orden de un proceso agregado $\mathrm{H}$-SSS escalan según una ley de potencia

$$
\operatorname{cum}_{m} X^{(n)}(k)=n^{m H_{s}} \operatorname{Cum}_{m} X(k)
$$

Si un proceso estacionario cumple con (5) para todo $n, m \in \mathbb{N}$, entonces log $\left|\operatorname{cum}_{m} X^{(n)}(k)\right|$ se comporta de forma tal que sus valores escalan linealmente con los de $\log n$, con coeficientes $m H_{S}$ que son función lineal de $m$. En otras palabras $m H_{S}=m H(m)$, es decir

$$
\operatorname{cum}_{m} X^{(n)}(k)=n^{m H(m)} \operatorname{cum}_{m} X(k)
$$

En [5] se demuestra que una generalización de un proceso autosimilar a un proceso multifractal es la siguiente: un proceso estacionario $X(k), k \in \mathbb{Z}$, es un proceso multifractal si $\log \left|\operatorname{cum}_{m} X^{(n)}(k)\right|=m H(m) \log n+c(m), \forall m, n \in \square$

permitiendo que el exponente $H$ varíe con el orden $m$.

La forma general para $m H(m)$ está dada por $m H(m)=\alpha m+\beta$

que corresponde al modelo fractal lineal, donde los coeficientes $\alpha$ y $\beta$ se determinan durante el ajuste de los cumulantes. En [8], [9] se demuestra que el único proceso conocido de este tipo tiene la forma

$$
m H(m)=m+2\left(H_{U}-1\right)
$$

y es llamado modelo unifractal.

En [5] se compara el modelo unifractal con el modelo autosimilar utilizando flujos empíricos. El análisis de las Figuras 3 y 4 de [5] coloca de manifiesto que ambos modelos capturan las principales tendencias de los flujos en la estimación de $H$, pero ninguno de ellos armoniza perfectamente con el fenómeno de localidad del exponente de Hurst.

La Figura 1 ilustra el fenómeno de localidad del exponente de Hurst. Se observa que la pendiente de la curva de ajuste cruza de un valor pequeño a uno notablemente mayor. Por lo tanto la curva se compone de tres partes: un segmento lineal con una pendiente suave cuando $\log n$ es pequeño, una pendiente intermedia, y un segmento lineal con una pendiente más pronunciada cuando log $n$ es grande.

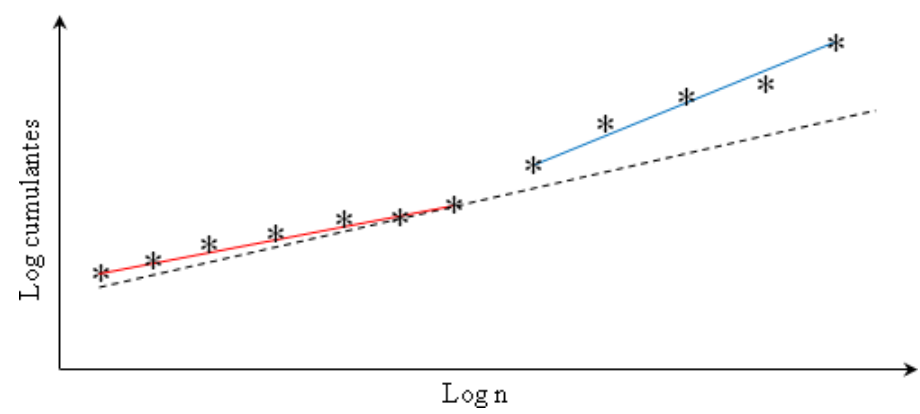

Figura 1: Fenómeno de localidad del exponente $H$.

\section{Un modelo multifractal que explica el fenómeno de localidad}

En [10] se reporta que el análisis de componentes principales del espectro de valores propios que resulta de mezclar señales de movimiento browniano fraccional con diferentes exponentes de Hurst, produce un comportamiento biescalar. Este preciso hecho es el que motiva el planteamiento de un modelo de flujo multifractal capaz de reproducir el fenómeno de localidad del exponente de Hurst. 
Suponiendo un proceso de flujo de tráfico de red $W(k)$, compuesto por dos procesos autosimilares independientes; $X_{1}(k)$ y $X_{2}(k)$, con exponentes de Hurst $H_{1}$ y $H_{2}$, respectivamente, es decir

$W(k)=\alpha_{1} X_{1}(k)+\alpha_{2} X_{2}(k)$

donde $\operatorname{Var}\left\{X_{1}\right\}=\operatorname{Var}\left\{X_{2}\right\}$ y los coeficientes de escala $\alpha_{1}$ y $\alpha_{2}\left(\alpha_{1}, \alpha_{2}>0\right)$ controlan la varianza de las componentes de (10). Además, sin pérdida de generalidad que $H_{1}<H_{2}$. Al igual que para (2), se define el proceso agregado $Z^{(n)}(k)$ a través de la multiplexación de incrementos $Z(k)=W(k+1)-W(k)$ considerando bloques no superpuestos de tamaño $n$ como

$Z^{(n)}(k)=\sum_{j=0}^{n-1} X(k n-j), \quad k \in \Pi, n \in \Pi$

Por independencia, para los modelos autosimilar y unifractal se verifica que

$\left|\operatorname{cum}_{m} Z^{(n)}(k)\right|=$

$\left|\operatorname{cum}_{m}\left(\alpha_{1} X_{1}^{(n)}(k)+\alpha_{2} X_{2}^{(n)}(k)\right)\right|$

es decir,

$\left|\operatorname{cum}_{m} Z^{(n)}(k)\right|=$

$c_{1}(m) n^{n H_{1}(m)}+c_{2}(m) n^{n H_{2}(m)}=$

$C_{1}(m) n^{m+2\left(H_{1}(m)-1\right)}+C_{2}(m) n^{m+2\left(H_{2}(m)-1\right)}$

con $c_{1}(m)$ y $c_{2}(m)$ parcialmente determinados por los coeficientes $\alpha_{1}$ y $\alpha_{2}$, respectivamente.
Luego, si $c_{1}(m)>c_{2}(m)$ existe una única solución positiva $n^{*}$ (que no es necesariamente entera) para la ecuación en términos de la variable $n$

$C_{1}(m) n^{m+2\left(H_{1}(m)-1\right)}=C_{2}(m) n^{m+2\left(H_{2}(m)-1\right)}$

Así, resulta fácil verificar que

$\left|\operatorname{cum}_{m} Z^{(n)}(k)\right|=$

$\left\{\begin{array}{l}C_{1}(m) n^{m+2\left(H_{1}(m)-1\right)}, \text { para } n \square n^{*} \\ C_{2}(m) n^{m+2\left(H_{2}(m)-1\right)}, \text { para } n \square n^{*}\end{array}\right.$

Para cada $m$ el diagrama en escala logarítmica del cumulante de $m$-ésimo orden de $Z^{(n)}(k)$ consiste en tres segmentos: un primer segmento con pendiente $m+2\left(H_{1}-1\right)$ cuando $n$ es pequeño; una sección de transición intermedia (muy corta) y, finalmente, un segmento lineal con pendiente $m+2\left(H_{2}-1\right)$ cuando $n$ es grande. Por lo tanto, se coloca en evidencia el fenómeno de localidad del exponente de Hurst.

La Figura 2 expone el fenómeno de localidad del exponente de Hurst para una serie FGN generada espectralmente [11] con $H=0,6$, mientras que la Figura 3 lo hace para una serie $F G N$ con $H=0,8$ también generada espectralmente. Finalmente, la Figura 4 muestra el comportamiento conjunto de ambas series considerando $\alpha_{1}=\alpha_{2}$ en (10).

Con todo lo anterior se demuestra de una manera simple que si un proceso de flujo de tráfico se forma a partir de dos componentes autosimilares aditivos independientes con exponentes de Hurst diferentes, entonces se observa el fenómeno de localidad en la estimación del exponente $H$ empleando cumulantes. No resulta difícil apreciar que el modelo propuesto puede generalizarse a más de dos componentes con similares resultados. 
Revista ECIPerú

Volumen 11, número 1

Octubre 2014

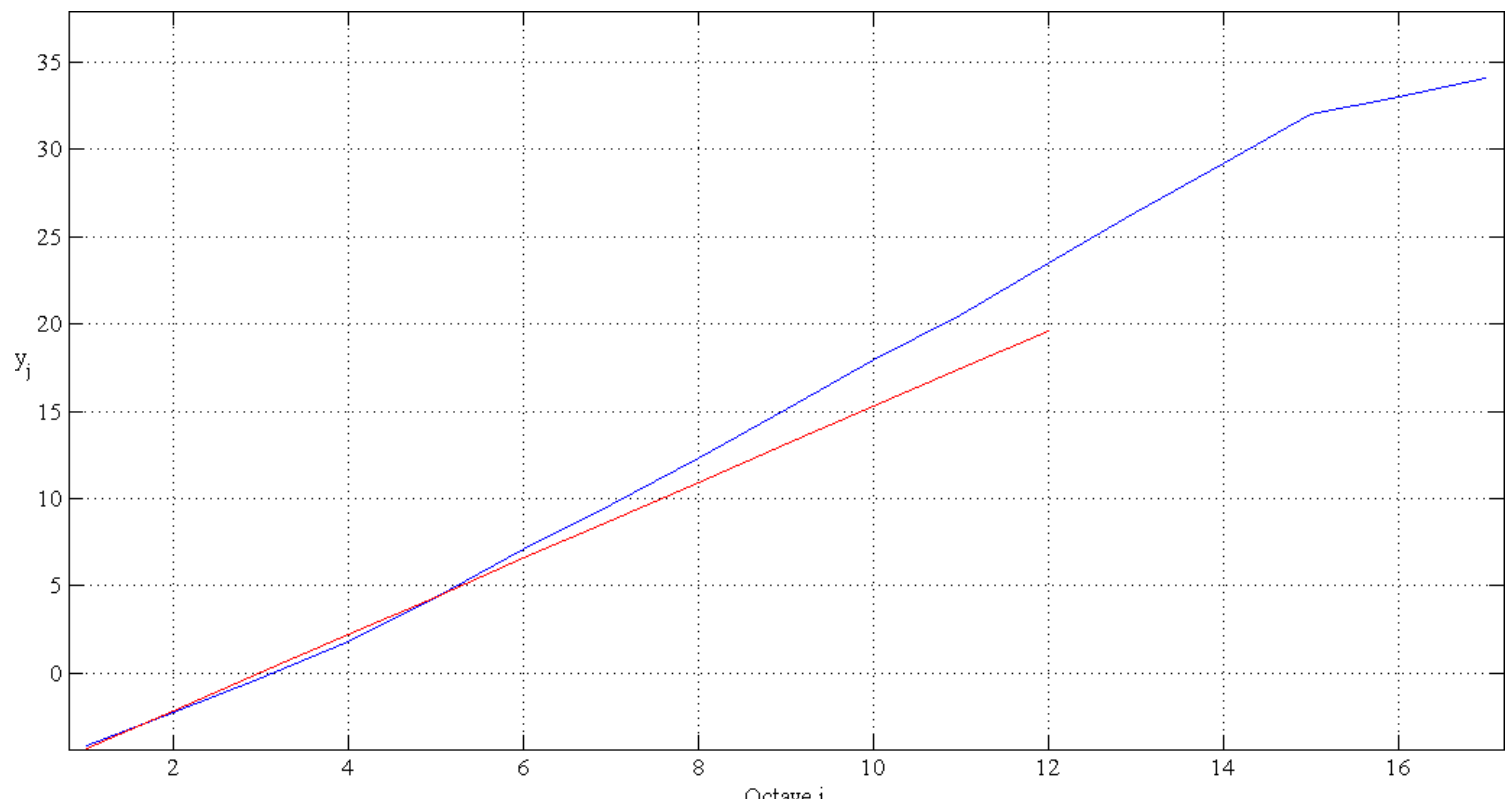

Figura 2: Fenómeno de localidad en una serie $F G N$ con $H=0,6$ generada espectralmente.

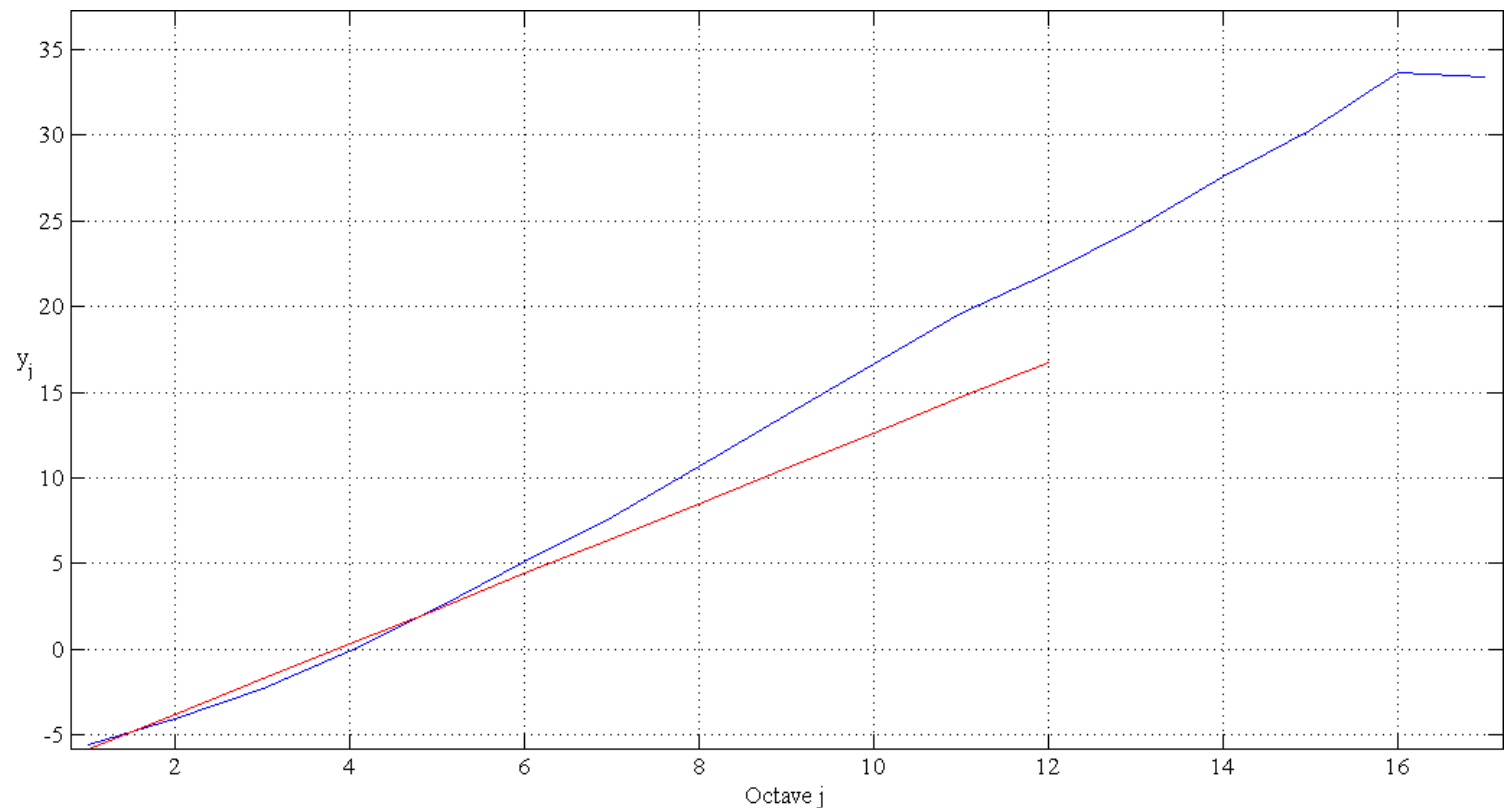

Figura 3: Fenómeno de localidad en una serie $F G N$ con $H=0,8$ generada espectralmente. 


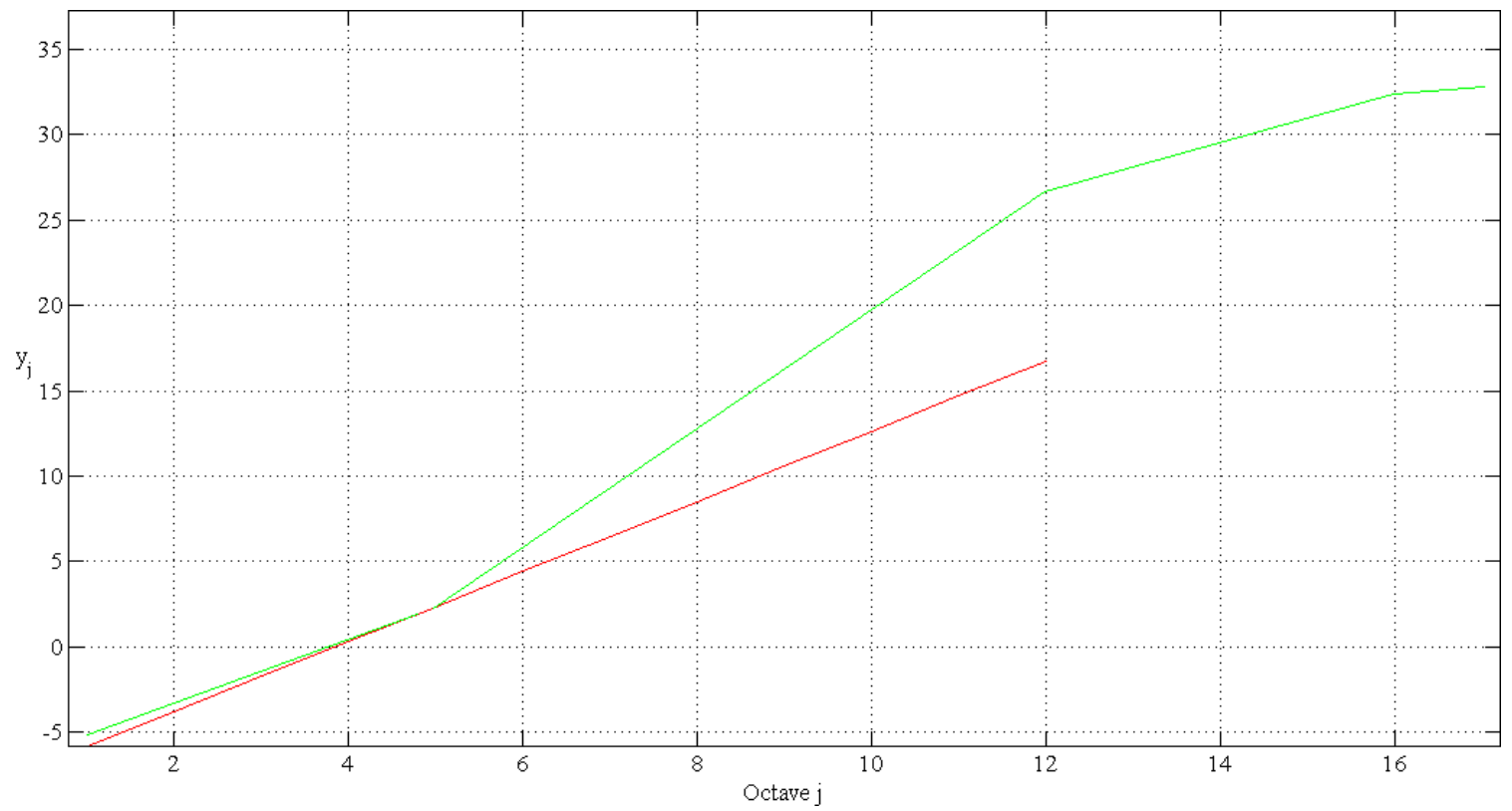

Figura 4: Fenómeno de localidad observado en el comportamiento conjunto de las series anteriores.

\section{Conclusiones}

Resulta interesante observar que la inconsistencia teórica de un único valor del exponente de Hurst y el fenómeno de localidad, pueden resolverse a través de un simple modelo que adiciona series temporales autosimilares de segundo orden. Por otra parte, es posible generar varios procesos con diferentes $H$, empleando para ello cualquiera de los métodos existentes para la generación de series autosimilares de segundo orden y luego adicionarlos. Esto está de acuerdo con la conclusión de [12], que establece que es necesario multiplexar diversas fuentes de tráfico para generar simulaciones más apropiadas para los flujos de tráfico.

La mayoría de los modelos anteriores que tratan los orígenes de la autosimilitud de los flujos de tráfico en las redes de alta velocidad, pueden ser adoptados, desde la perspectiva de su aplicación al modelo propuesto, con el objetivo de recalcar la coexistencia en los flujos reales, de múltiples componentes autosimilares heterogéneos. Puede afirmarse que si en el modelo On/Off de [13] se supone la existencia de dos valores diferentes para los exponentes de Pareto que controlan las distribuciones los periodos On y los periodos Off de las fuentes, entonces el proceso de flujo final agregado presentará dos componentes diferentes en términos de sus exponentes de Hurst y, por lo tanto, dará cuenta de la existencia del fenómeno de localidad.

En general, para redes de grandes coberturas, es decir con estructuras muy complejas, el canal de datos es compartido por múltiples fuentes en una forma que puede considerarse aproximadamente independiente y aditiva. Debido a la diversidad de las fuentes y los mecanismos de transferencia, el flujo que ingresa proveniente de cada fuente posee diferentes exponentes de Hurst. Esto lleva a plantear la siguiente conjetura: es más probable observar el fenómeno de localidad en flujos de tráfico de redes troncales que locales.

\section{Referencias}

[1] G. Millán, H. Kaschel, and G. Lefranc. "Discussion of the analysis of self-similar teletraffic with long-range dependence (LRD) at the network layer level". Int. J. Comput. Commun. Vol. V № 5, pp. 799-812. December 2010.

[2] Y.-D. Chen, L. Li, Y. Zhang, and J.-M. Hu. "Fluctuations and pseudo long range dependence in network flows: a non-stationary Poisson process model". Chinese Physics B. Vol. 18 № 4, pp. 1373-1379. April 2009.

[3] G. Millán, H. Kaschel, and G. Lefranc. "A simple model for the generation of LRD selfsimilar traffic using piecewise affine chaotic one-dimensional maps". Stud. Inform. Control. Vol. 19 № 1, pp. 67-78. March 2010.

[4] J. G. Evertsz and B. B. Mandelbrot. "Multifractal measures". In Chaos and Fractals, H.-O. Peitgen, H. Jürgens, and D. Saupe, Eds. 1st Ed. Springer-Verlag, New York, Appendix B, pp. 849-881, 1992.

[5] G. Terdik and T. Gyires. "Lévy flights and fractal modeling of Internet traffic". IEEE/ACM Trans. Netw. Vol. 17 № 1, pp. 120-129. Feb. 2009.

[6] O. I. Sheluhin, S. M. Smolskiy, and A. V. Osin. "Self-Similar Processes in Telecommunications". 
Wiley. UK. 2007.

[7] G. Terdik. "Bilinear stochastic models and related problems of nonlinear time series analysis: a frequency approach". Lectures Notes in Statistics. Vol. 142. Springer-Verlag. New York. 1999.

[8] E. Iglói and G. Terdik. "Superposition of diffusions with linear generator and its multifractal limit process". ESAIM-Probab. Stat. Vol. 7, pp. 23-88. May 2003.

[9] S. Molnár and G. Terdik. "A general fractal model of Internet traffic". In Proc. 26th Annu. IEEE Conf. Local Computer Networks. Tampa, FL, pp. 492-499 2001.

[10] L. Li, J. Hu, Y. Chen, and Y. Zhang. "PCA based Hurst exponent for $\mathrm{fBm}$ signals under disturbances". IEEE Trans. Signal Process. Vol. 57 № 7, pp. 2840-2846. July 2009.

[11] P. Abry, P. Flandrin, M. S. Taqqu, and D.
Veitch. "Self-similarity and long-range dependence through the wavelets lens". In Theory and Applications of Long-Range Dependence, P. Doukhan, G. Oppenheim, and M. S. Taqqu, Eds. Birkhäuser, Boston, MA, pp. 527-556, 2003.

[12] G. Horn, A. Kvalbein, J. Blomskøld, and E. Nilsen. "An empirical comparison of generators for self-similar simulated traffic". Perform. Evaluation. Vol. 64 № 2, pp. 162-190. February 2007.

[13] W. Willinger, M. S. Taqqu, R. Sherman, and D. V. Wilson. "Self-similarity through highvariability: statistical analysis of Ethernet LAN traffic at the source level". IEEE/ACM Trans. Netw. Vol. 5 № 1, pp. 71-86. February 1997.

E-mail: gmillan@ucn.cl 